\title{
Computing Is the Secret Ingredient (well, not so secret)
}

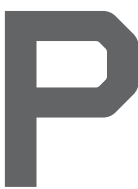

ERHAPS YOU REMEMBER the iconic theme of the globally popular Kung $\mathrm{Fu}$ Panda movies, "You are the secret ingredient!" This meant that self-belief is important and with it great things can be achieved-Po, for example, became the Dragon Warrior. My meaning here is that computer science is both a powerful enabler of rapid advances in all intellectual fields and a disruptor driving furious revolutions in commerce and society worldwide. Computer science is more important and potent than ever!

Computing is driving unprecedented rapid change. One cluster of revolutions is around artificial intelligence (AI) and machine learning. Every day brings evidence of the rapidly growing capabilities in AI, driven by a host of algorithmic advances, but notably machine learning, to perform tasks heretofore exclusively the province of humankind. With high-profile applications such as speech, question and answer systems, image and face recognition, robotics with growing autonomy and flexibility-including self-driving cars, deep ocean exploration, and space exploration-society is broadly aware of AI's growing capabilities. And worldwide, industry is ablaze in virtually every sector with the specter of disruptive, radical new opportunities.

Further, as AI capabilities push computing into new domains, there is growing concern ${ }^{2}$ in economic, policy, and computing communities about the potential impact on employment, types of work, and global competition.

A second cluster of revolution is around blockchain and decentralized trust. The advent of Bitcoin has produced a cascade of academic research, startup experimentation, industrial innovation, and now an explosion of change. An initial burst of innovation around electronic currencies that improve Bitcoin to create currencies with different properties continues to make waves, sparking new activity, government regulation, and in some cases outright bans. Over time, innovation has matured and broadened into a staggering breadth of applications based on the core disruptions that decentralized trust represents. Reformulated business and government activities around a distributed, trusted ledger abound, including new approaches to provenance, decentralized markets, and a host of financial applications, but few examples of the structural disruptions from centralized to distributed trust that blockchain is driving.

Equally exciting are efforts to recast foundational Internet services such as DNS on blockchain ideas. The Internet was conceived as the decentralized network of peers, but the design of core services, such as DNS, have always depended on trusted services - and organizations. Reinventing these services' decentralized trust presents a radical new future for these and perhaps many types of Internet services and distributed systems.

While these two clusters of revolutions are enabled by generations of Moore's Law and large-scale distributed systems, its notable that the essence of each is algorithmic advances and breakthroughs. Algorithms are in the essential core of the field of computer science.

A third revolution is the growing recognition that computer science is a fundamental element of secondary education. Recent signs include formal adoption of computer science education guidelines for primary and secondary education in the U.K., ${ }^{3}$ an ACMIndustry vision, ${ }^{1}$ and a $\$ 200 \mathrm{M}$ U.S. Department of Education program ${ }^{1}$ to promote high-quality STEM and computer science education curriculum and programs in high schools.

While these are just three, there are doubtless many more, and I would love to hear about them!

Computing is more important than ever and driving disruptive change. But it's also moving faster than ever because of so many creative and innovative computing professionals, huge capabilities in chips and massive clouds, and companies with extraordinary scope and ability to drive innovation. One of the giants of our field, the late Jim Gray, said in his 1999 Turing lecture, "Boy, we thought computing was moving fast in the 70's and 80's, but it's really moving fast now." I believe the rate of change that computer science is advancing more rapidly and driving. more change in society today ... and with dramatically broader scope.

So, sit back for a moment these holidays as a computing professional and revel in where we are, and think about the exciting opportunities - and responsibilities—we have before us!

Andrew A. Chien, EDITOR-IN-CHIEF

Andrew A. Chien is the William Eckhardt Distinguished Service Professor in the Department of Computer Science at the University of Chicago, Director of the CERES Center for Unstoppable Computing, and a Senior Scientist at Argonne National Laboratory.

\section{References}

1. ACM and Partners Release Framework for Computer Science Education in U.S. K-12 Schools, Oct. 18, 2016.

2. De Lange Conference on Humans, Machines, and the Future of Work, Dec. 5, 2016

3. United Kingdom Department of Education. Statutory Guidance: National Curriculum in England: Computing Programmes of Study, Sept. 11, 2013

4. U.S.A. Presidential Memorandum on Creating Pathways to Jobs by Increasing Access to High-Quality Science, Technology, Engineering, and Mathematics and Computer Science Education. Sept. 25, 2017.

Copyright held by author. 